\title{
Cambios en las propiedades morfológicas de suelos pardos sialíticos sometidos a diferentes manejos agrícolas en Cuba
}

\author{
Changes in the morphological properties of sialitic Brown soils \\ subjected to different agricultural management in Cuba. \\ Yoandris Socarrás Armenteros ${ }^{1 *}$, Alberto Hernández Jiménez ${ }^{2}$, Elein Terry Alfonso ${ }^{2}$, \\ Pedro José González Cañizares ${ }^{2}$, Ángel Lázaro Sánchez Iznaga1, Orlando Delgado Cabrera ${ }^{3}$
}

\section{RESUMEN}

\begin{abstract}
La investigación se desarrolló en la finca El Aeropuerto ubicada en el municipio Cienfuegos, en Cuba, con el objetivo de diagnosticar los cambios de las propiedades morfológicas de un suelo pardo sialítico formado de una arcilla calcárea del Oligoceno. Se analizaron las propiedades morfológicas y químicas del suelo bajo tres manejos diferentes: 1: bosque, 2: conservado, 3: agrogénico. Los métodos analíticos utilizados fueron textura por el triángulo textural, materia orgánica, $\mathrm{pH}$, cationes intercambiables, fósforo asimilable. Como resultado en la caracterización morfológica de cada perfil, se presentó el horizonte mullido en el suelo patrón, propiedades eslíticas en el suelo conservado y vérticas en el suelo sometido a cultivo intensivo y continuado (agrogénico). Todo parece indicar que la evolución del suelo y el empeoramiento de sus propiedades están en relación directa con el manejo a que ha sido sometido. El pH va de ligeramente alcalino a alcalino. La suma de bases cambiables es alta, sobre todo en la parte superior del perfil de pasto y cultivos intensivos, y muy alta en el horizonte superior del perfil de bosque. El perfil de área bosque tiene mayor contenido de materia orgánica.
\end{abstract}

Palabras clave: edafología, perfil diagnóstico, degradación propiedades.

\section{ABSTRACT}

The research was carried out at the El Aeropuerto farm located in the Cienfuegos municipality in Cuba, with the objective of diagnosing the changes in the morphological properties of a sialitic Brown soil formed of Oligocene calcareous clay. The morphological and chemical properties of the soil were analyzed under three different managements: 1: forest, 2: conserved, 3: agrogenic. The analytical methods used were: textural triangle texture, organic matter, $\mathrm{pH}$, interchangeable cations, assimilable phosphorus. As a result of the morphological characterization of each profile, the fluffy horizon was presented in the pattern soil; the soil is conserved in the soil and the soil in the soil subjected to intensive and continuous (agrogenic) cultivation. The worsening of its properties is in direct relation with the management to which it has been subjected. The pH ranges from slightly alkaline to alkaline. The sum of changeable bases is high, especially in the upper part of the grass profile and intensive crops, and very high in the upper horizon of the forest profile. The forest area profile has a higher content of organic matter.

Keywords: edaphology, diagnostic profile, degradation properties.

El hombre, a través de los siglos, ha sido el principal factor alterador del medio ambiente. Desgraciadamente, la mayoría de esas modificaciones están relacionadas con altas tasas de deforestación y degradación de suelos.

La degradación de los suelos es un proceso complejo, en el cual varios factores naturales o inducidos por el hombre contribuyen a la pérdida de su capacidad productiva. Indiscutiblemente, la degradación es un problema global que amenaza directamente a más de 250 millones de personas y cerca de 4 mil millones de hectáreas de tierras agrícolas que han perdido su capacidad agroproductiva. En el mundo, aproximadamente el

\footnotetext{
1 Universidad de Cienfuegos, Carretera Roda, Km 1 Cuatro Caminos. Cienfuegos. Universidad de Cienfuegos. Convenio de colaboración entre la Universidad de Cienfuegos (UCF) y la Universidad Metropolitana de Ecuador (UMET). Quito, Ecuador.

2 Instituto Nacional de Ciencias Agrícolas, Carretera Tapaste, Km 31/2. San José de las Lajas, Mayabeque.

3 Empresa de Aprovechamiento Hidráulico Cienfuegos.

* Autor para correspondencia: ysocarras@ucf.edu.cu
}

Fecha de Recepción: 21 Noviembre, 2018.

Fecha de Aceptación: 10 Mayo, 2019. 
70\% de los 5. 200 millones de hectáreas de tierras secas que se utilizan para la agricultura están degradadas y amenazadas (Arias et al., 2010a).

Cuba no es ajena a esta problemática. El área agraria es de 6,7 millones de hectáreas y según el Instituto de Suelos, el 76,89\% de esa superficie está afectada por algún factor que limita su productividad. Por ello están considerados como suelos poco productivos. El $43 \%$ sufre erosión, el $14 \%$ compactibilidad, el $70 \%$ con bajo contenido de materia orgánica, el $14 \%$ con salinidad, el $37 \%$ baja retención de humedad, el $45 \%$ baja fertilidad, el $12 \%$ pedregosidad y el $40 \%$ tiene drenaje deficiente, según reporta Muñiz (2017).

La Estrategia Ambiental Nacional, para los períodos 2000-2005 y 2006-2010, identificó la degradación de suelos como uno de los cinco problemas ambientales principales de Cuba (CITMA, 2000).

En Cuba los suelospardos ocupan una superficie de 2.356000 ha $(27,03 \%)$ y se desarrollan en relieves llanos a ondulados. Son los suelos más extensos del país y están representados en todas las provincias, aunque son más importantes en las centrales y orientales (Arias et al., 2010b). En el caso específico de Cienfuegos, existe un total de 143,8 (Mha) de suelos pardos sialíticos, lo que representa el $30 \%$ del total de los suelos agrícolas de la provincia. La mayoría de estos suelos están dedicados a cultivos varios y caña de azúcar.

Tomando en cuenta estos antecedentes, el presente trabajo tuvo como objetivo general Diagnosticar los cambios de las propiedades morfológicas de un suelo pardo sometido a diferentes formas de manejo.

\section{Materiales y Métodos}

La investigación se realizó en la finca El Aeropuerto ubicada en la provincia y municipio Cienfuegos de Cuba, en el período de junio - julio de 2016.

Para el diagnóstico se tomaron tres perfiles de suelos pardos en diferentes condiciones de manejo. Los perfiles estudiados en relación con el uso del suelo se presentan a continuación:

Perfil 1: Tomado bajo bosque (> 50 años) (suelo patrón o de referencia).

Perfil 2: Tomado en un área de cultivo continuado (suelo agrogénico).

Perfil 3: Tomado en un área de pasto (10-15 años) (suelo conservado).
La clasificación y definición de cada suelo corresponde a la utilizada por Hernández et al., (2015). La descripción de los perfiles se realizó por el Manual para la Cartografía Detallada y Evaluación Integral de los suelos (Hernández et al., 1995). Se presenta además la correlación de los perfiles estudiados con el World Reference Base (IUSS Working Group WRB, 2014) y Soil Taxonomy (Soil Survey Staff, 2010).

El ecosistema en estudio cuenta con las siguientes características:

Material de origen: arcilla calcárea en perfiles 1 y 3 , arenisca sin carbonatos en el perfil 2.

Clima: tropical subhúmedo Precipitaciones anuales: $1.400-1.450 \mathrm{~mm}$.

Temperatura media anual: $24-24,5^{\circ} \mathrm{C}$.

\section{El estado actual de los suelos se determinó a través del análisis de los siguientes indicadores:}

\section{Características morfológicas y químicas}

- Tipos de horizontes genéticos.

- Tipos de horizontes y características de diagnóstico.

- Transición entre los horizontes.

- Textura por el triángulo textural.

- Consistencia.

- Porosidad.

- Reacción al HCl.

- Materia orgánica (\%) por el método Walkley - Black (Combustión Humedad).

- $\mathrm{pH}$, por potenciometría relación suelo: agua 2,5: 1 .

- Cationes intercambiables por el método con AcNH4.

- Fósforo asimilable por Oniani.

- El color en las descripciones del suelo se determinó por la Tabla de Colores Munsell (Munsell Soil Color Chart, 2009).

\section{Resultados y Discusión}

\section{Características morfológicas de los suelos}

La morfología de los perfiles de suelos brinda una información muy importante para diagnosticar los procesos de formación y propiedades de los suelos que se derivan de estos procesos. Esta es la primera 
información que debe enfrentar el edafólogo en sus investigaciones (Gueraimova y Khitrov, 2016).

Además, se ha demostrado que el manejo diferenciado de los suelos, sobre todo en la variante de cultivo intensivo y continuado, se manifiesta inicialmente en las características morfológicas de los suelos, como se plantea en los resultados obtenidos para los suelos ferralíticos rojos lixiviados de las llanuras cársicas (Hernández et al., 2013, 2014).

Como se observa en la Tabla 1, este perfil de suelo bajo bosque secundario se forma bajo el proceso de sialitización, por el color pardo amarillento oscuro y estructura de bloques, típico de los suelos con predominio de arcillas del tipo 2:1.

Este proceso permite la clasificación de los suelos en el agrupamiento de suelos pardos sialíticos y en el tipo genético pardo. En el horizonte A y $\mathrm{AB}$ se presenta una estructura granular nuciforme, que permite diagnosticar el horizonte secundario mullido. Además, por este tipo de estructura y el color bien oscuro, es propio del proceso de humificación, que diagnostica el horizonte secundario humificado. Asimismo, en el perfil se detecta que no tiene carbonatos en el horizonte $\mathrm{A}$ ni en el B. Por tanto, acorde a la clasificación de Suelos de Cuba 2015 (Hernández et al., 2015) tiene un perfil diagnóstico Amh-Bsial-Ck (Figura 1). Suelos de Cuba (2015): pardo mullido y húmico, lavado; World Reference Base (2014): Feozem cámbico, calcárico, arcilloso; Soil Taxonomy (2010): Typic Haplustoll.

La degradación física en la cuenca se manifiesta principalmente en el deterioro de la estructura

Tabla 1. Características morfológicas de los perfiles con diferentes formas de manejo.

\begin{tabular}{|c|c|c|c|c|c|}
\hline Horizontes & $\begin{array}{l}\text { Profundidad } \\
\quad(\mathrm{cm})\end{array}$ & $\begin{array}{l}\text { Clase } \\
\text { textural }\end{array}$ & Estructura & Color & Consistencia \\
\hline \multicolumn{6}{|c|}{ Perfil de bosque } \\
\hline $\mathrm{A}_{11 \mathrm{~m}}$ & $0-13$ & Arcillosa & Nuciforme y granular & P. grisáceo muy oscuro (10YR3/2) & Friable \\
\hline $\mathrm{A}_{12 \mathrm{~m}}$ & $13-25$ & Arcillosa & B. angulares & P. grisáceo oscuro (10YR4/2) & Compactado y plástico \\
\hline $\mathrm{B}$ & $25-40$ & Arcillosa & $\begin{array}{l}\text { B. subangulares y } \\
\text { angulares }\end{array}$ & P. amarillento oscuro (10YR4/4) & Compactado y algo plástico \\
\hline $\mathrm{BC}$ & $40-60$ & $\begin{array}{l}\text { Franco } \\
\text { arcilloso }\end{array}$ & B. angulares & P. amarillento (10YR5/6) & Friable \\
\hline $\mathrm{C}_{\mathrm{k}}$ & $60-+$ & Franco & B. angulares & Amarillo pardusco (10YR6/6) & Friable \\
\hline \multicolumn{6}{|c|}{ Perfil de cultivo } \\
\hline $\mathrm{A}_{11} \mathrm{SL}$ & $0-18$ & Arcillosa & $\begin{array}{l}\text { B. subangulares y } \\
\text { angulares }\end{array}$ & Gris muy oscuro (10YR3/1) & Compactado y plástico \\
\hline $\mathrm{A}_{12} \mathrm{SL}$ & $18-42$ & Arcillosa & B. prismática & Gris muy oscuro (10YR3/1) & Compactado y algo plástico \\
\hline $\mathrm{B}_{1}$ & $42-60$ & Arcillosa & B. prismáticos & $\begin{array}{l}\text { P. amarillento oscuro, con manchas P. } \\
\text { grisáceo muy oscuro (10YR4/6, 10YR3/2m) }\end{array}$ & Plástica \\
\hline $\mathrm{B}_{2(\mathrm{~g})}$ & $60-90$ & $\begin{array}{l}\text { Franco } \\
\text { arcilloso }\end{array}$ & no apreciable & $\begin{array}{l}\text { P. amarillento, con manchas rojo amarillento } \\
\text { y oscuras (10YR5/6, 5YR4/6) }\end{array}$ & Plástica \\
\hline \multicolumn{6}{|c|}{ Perfil de pasto } \\
\hline $\mathrm{A}_{11}$ & $0-25$ & Arcillosa & B. subangulares & P. grisáceo oscuro (10YR4/2) & $\begin{array}{l}\text { Ligeramente compactado y } \\
\text { plástico }\end{array}$ \\
\hline $\mathrm{B}$ & $25-45$ & Arcillosa & $\begin{array}{l}\text { B. subangulares algo } \\
\text { prismática }\end{array}$ & P. amarillento oscuro (10YR3/4) & Compactado y plástico \\
\hline $\mathrm{BC}$ & $45-60$ & Arcillosa & B. subangulares & P. amarillento (10YR5/6) & Compactado y plástico \\
\hline $\mathrm{C}$ & 60 & $\begin{array}{l}\text { Franco } \\
\text { arcilloso }\end{array}$ & $\begin{array}{l}\text { B. subangulares y } \\
\text { angulares }\end{array}$ & Amarillo pardusco (10YR6/6) & Friable \\
\hline
\end{tabular}

P: Pardo; B: Bloque. 

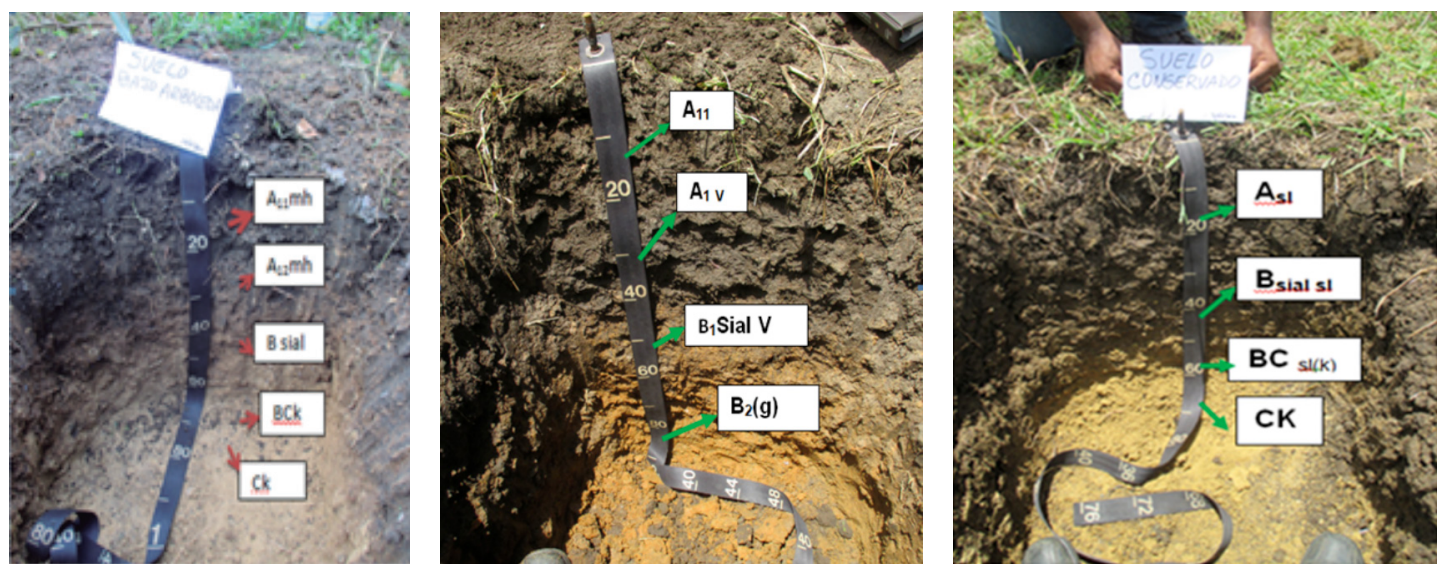

Figura 1. Perfil diagnóstico de cada área con diferente forma de manejo.

natural del suelo como resultado de un uso agrícola intensivo (Torres et al., 2003). La degradación asociada al cambio de uso de suelo provoca una disminución significativa en la materia orgánica, la capacidad de intercambio catiónico de los suelos destinados al cultivo, así como la pérdida de más del 55\% del carbono secuestrado (Cruz et al., 2012). Los sistemas forestales secundarios demuestran un uso más sostenible, con buena resistencia después de nueve años de barbecho (Melo et al., 2017a).

Por la descripción del perfil 2, se observa que el suelo se ha formado por el proceso de sialitización, dando lugar al horizonte principal de diagnóstico siálico, por lo que el suelo es del tipo pardo, pero a diferencia del perfil 1, no es ni mullido ni húmico. Presenta una estructura de bloques prismáticos entre los 18 y $60 \mathrm{~cm}$ de profundidad, con caras de deslizamiento, dando lugar a las características vérticas. En el horizonte $B_{2}$, se diagnostican manchas de gleyzación suave. No tiene reacción al ácido clorhídrico en todo el perfil, por lo que no hay presencia de carbonato de calcio.

El suelo se clasifica como pardo vértico y gléyico, sin carbonatos, con un perfil diagnóstico del tipo A-B $\mathbf{B}_{1}$ sialv-B $\mathbf{B}_{2} \operatorname{sial}(\mathbf{g}$ ) (Figura 1). Suelos de Cuba (2015): pardo eslítico y gléyico, sin carbonatos; World Reference Base (2014): Feozem gléyico, vértico, cámbico, ánthrico, arcilloso; Soil Taxonomy (2010): Vertic Haplustoll.

Como puede observarse por la descripción, el perfil también está formado bajo el proceso de sialitización, con el tipo genético de suelo pardo. Muy importante resulta el tipo de estructura, que no llega a ser prismática, lo cual evidencia que no tiene características vérticas.
El término eslítico viene del ruso "slitii", que quiere decir compacto-plástico. En este caso, no se ven bien conformadas las caras de deslizamiento. Es decir, existe en un estadio de formación de suelos antes del subtipo vértico.

La presencia del horizonte eslítico como subtipo de suelo pardo fue destacada hace algunos años en Cuba (Morales et al., 2005). Es aplicado por primera vez por Hernández 2015 y Hernández et al., 2015. En este perfil, de acuerdo con la Clasificación de Suelos de Cuba 2015, se estaría en presencia de propiedades eslíticas, por lo que el suelo a nivel de subtipo es pardo eslítico.

$\mathrm{Al}$ igual que el perfil bajo bosque secundario, este suelo está formado a partir de arcilla calcárea y el perfil en los horizontes A y B no tiene carbonatos, por lo que se corresponde con el género lavado.

$\mathrm{Al}$ analizar las características morfológicas de estos suelos, se observan diferencias notables, para lo cual resulta muy importante diagnosticar con precisión la estructura del suelo. El tipo de estructura del suelo es una de las características fundamentales, ya que está en correspondencia con el proceso de formación del suelo y la antropogénesis. Según Hernández et al., 2010, resulta uno de los indicadores más importantes del estado actual del suelo y permite establecer un diagnóstico adecuado.

Todo parece indicar que con la antropogénesis cambia completamente el suelo. En primer lugar, se pierde el horizonte mullido y se pasa a la eslitización y posteriormente a la formación de características vérticas. Es posible que en condiciones de bosques, el suelo mantenga una humedad todo el año en los límites de la humedad productiva y que cuando se 
elimina el bosque y se pasa a cultivo, aumenta la evapotranspiración, lo que trae consigo la ascensión capilar de la humedad del suelo.

La ascensión capilar es más rápida en la parte superior del suelo y más lenta desde la parte media e inferior, llegando a los límites de la humedad de ruptura capilar. Con ello comienzan a individualizarse los agregados en pequeños prismas, pasando a la estructura prismática, y con el tiempo se forman las caras de deslizamiento. Este proceso descrito por Agafonov en su tesis doctoral, para los vertisoles de Cuba, ocurre en estos suelos, pero en ellos los bloques prismáticos no son tan grandes debido principalmente al espesor arcilloso, medianamente profundo.

Los sistemas de labranza modifican la estructura del suelo y, dependiendo del contenido de humedad de este, la labranza favorece o destruye la estructura, repercutiendo en el crecimiento y desarrollo de los cultivos (Navarro et al., 2000).

Morrell et al. (2008) demostraron que a medida que va siendo más intensa la acción antrópica, mayores son las pérdidas en la estructura de los suelos, hasta un punto que lleva a la degradación de estos, así como a la pérdida en sus contenidos en materia orgánica, nutrientes para las plantas.

La principal causa de degradación física del suelo es la compactación, provocada por presión sobre el suelo (González et al., 2009). Se observó que en los suelos cambisoles, solonetz y arenosoles hay modificaciones en la estructura del suelo, por el cultivo continuado, formándose bloques en los primeros $20 \mathrm{~cm}$, que alcanzan un tamaño de aproximadamente $20 \mathrm{~cm}$ o más (Murray et al., 2015).

En el perfil 3, que estuvo bajo caña de azúcar por más de 60 años, la intensidad del proceso es menos marcada, ya que solamente hay inicios de la formación de bloques prismáticos y casi no hay cara de deslizamiento. Esto pudo deberse a que el cultivo de la caña resulta una plantación que cuando cubre el suelo la evapotranspiración no es tan fuerte, además de que hace 10 años el uso del suelo cambió a pastizal.

Estas transformaciones demuestran que realizar una interpretación adecuada de las características morfológicas del suelo es muy útil para el edafólogo, ya que como plantea la escuela tradicional rusosoviética, "el perfil habla".

La clasificación de los Suelos de Cuba (2015): pardo eslítico, lavado y su perfil diagnóstico es Asl-Bsialsl-Ck (Figura 1). Pardo eslítico, lavado; World Reference Base (2014): Feozem cámbico, ánthrico, calcárico, arcilloso; Soil Taxonomy (2010): Udertic Haplustoll.

Senjobi y Ogunkunle (2011) expresaron que existe la necesidad de comprender el suelo adecuadamente a través de un estudio detallado

Tabla 2. Propiedades fisico químicas de un suelo pardo bajo diferentes formas de manejo en la provincia Cienfuegos.

\begin{tabular}{|c|c|c|c|c|c|c|c|c|c|}
\hline \multirow{2}{*}{ Horizonte } & \multirow{2}{*}{$\begin{array}{l}\text { Profundidad } \\
\quad(\mathrm{cm})\end{array}$} & \multirow{2}{*}{$\begin{array}{c}\mathrm{pH} \\
\left(\mathrm{H}_{2} \mathrm{O}\right)\end{array}$} & \multirow{2}{*}{$\begin{array}{l}\text { MO } \\
(\%)\end{array}$} & \multirow{2}{*}{$\begin{array}{c}\mathrm{P} \\
(\mathrm{ppm})\end{array}$} & $\mathrm{Ca}$ & $\mathrm{Mg}$ & $\mathrm{Na}$ & $\mathrm{K}$ & \multirow{2}{*}{ Suma } \\
\hline & & & & & \multicolumn{4}{|c|}{$\left(\mathrm{cmol} \mathrm{kg}^{-1}\right)$} & \\
\hline \multicolumn{10}{|c|}{ Perfil de bosque } \\
\hline $\mathrm{A}_{11 \mathrm{~m}}$ & $0-13$ & 7,6 & 5,31 & 490,6 & 33,0 & 10,0 & 0,25 & 1,01 & 44,26 \\
\hline $\mathrm{A}_{12 \mathrm{~m}}$ & $13-25$ & 8,0 & 3,23 & 158,0 & 28,5 & 10,5 & 0,22 & 0,59 & 39,81 \\
\hline B & $25-40$ & 8,0 & 1,93 & 31,87 & 28,5 & 7,00 & 0,17 & 0,41 & 36,08 \\
\hline $\mathrm{BC}$ & $40-60$ & 8,1 & 1,67 & 1,95 & 23,5 & 11,0 & 0,15 & 0,41 & 35,06 \\
\hline $\mathrm{C}_{\mathrm{k}}$ & $60-+$ & 8,2 & 1,38 & 21,70 & 17,5 & 4,50 & 0,15 & 0,28 & 22,43 \\
\hline \multicolumn{10}{|c|}{ Perfil de cultivo } \\
\hline $\mathrm{A}_{11} \mathrm{SL}$ & $0-18$ & 7,8 & 2,62 & 48,4 & 26,5 & 9,00 & 0,46 & 0,50 & 36,46 \\
\hline $\mathrm{A}_{12} \mathrm{SL}$ & $18-42$ & 7,9 & 2,55 & 33,7 & 23,0 & 12,0 & 0,57 & 0,38 & 35,95 \\
\hline $\mathrm{B}_{1}$ & $42-60$ & 8,4 & 1,97 & 8,83 & 23,5 & 5,00 & 0,84 & 0,36 & 29,70 \\
\hline $\mathrm{B}_{2(\mathrm{~g})}$ & $60-90$ & 8,4 & 1,06 & 22,60 & 24,0 & 1,00 & 1,36 & 0,37 & 26,73 \\
\hline \multicolumn{10}{|c|}{ Perfil de pasto } \\
\hline $\mathrm{A}_{11}$ & $0-25$ & 7,6 & 3,00 & 32,60 & 44,5 & 10,0 & 0,13 & 0,62 & 55,25 \\
\hline B & $25-45$ & 7,9 & 2,78 & 30,90 & 51,0 & 2,00 & 0,24 & 0,56 & 53,80 \\
\hline $\mathrm{BC}$ & $45-60$ & 8,2 & 1,71 & traza & 39,5 & 9,00 & 0,63 & 0,45 & 49,58 \\
\hline $\mathrm{C}$ & 60 & 8,2 & 0,96 & 20,00 & 27,5 & 9,00 & 0,90 & 0,36 & 37,76 \\
\hline
\end{tabular}


de este y una evaluación de la tierra, con el fin de utilizarla de manera apropiada para los cultivos.

En la Tabla 2 se muestran las características fisicoquímicas del suelo de cada perfil. Teniendo en cuenta los resultados, se puede observar que en los tres perfiles estudiados el $\mathrm{pH}$ va de ligeramente alcalino a alcalino, y es más alcalino en profundidad por la presencia de los carbonatos.

Estos valores se asemejan a los de Melo et al. (2017b), según los cuales en áreas cultivadas y bosques secundarios, los valores de $\mathrm{pH}$ alcanzaron valores más altos que en los bosques nativos.

La suma de bases cambiables es alta, sobre todo en la parte superior del perfil de pasto y cultivos intensivos, y muy alta en el horizonte superior del perfil de bosque. En este resultado puede incidir la alta presencia de cationes de calcio y magnesio, y, al mismo tiempo, la presencia de arcillas del tipo de la montmorillonita, lo que demuestra el proceso de sialitización, dando lugar a la formación de suelos pardos sialíticos, con el tipo de suelo pardo.

El contenido de materia orgánica $(\mathrm{MO})$ varía para cada perfil de suelo. Existe alto contenido de MO en el horizonte superior y muy bajo en los horizontes inferiores para el perfil bosque. En los perfiles de cultivo y pastos existe poca presencia de materia orgánica en los horizontes evaluados. No obstante, es ligeramente superior al perfil de suelo denominado "conservado", bajo pastos.

Una de las principales causas del bajo contenido de materia orgánica en el suelo en los perfiles de cultivo y pastos es el sistema de monocultivo con caña de azúcar por más de 60 años, y a partir de 1997 esos suelos cambiaron su forma de manejo para pastos y cultivos varios. Para el caso del área de cultivos varios, diez años atrás se realizaron aplicaciones de compost como enmienda orgánica.

Estos resultados se relacionan con los obtenidos por Domínguez et al. (2006), los cuales identificaron como causas, la no aplicación sistemática de enmendantes orgánicos y la poca protección y aplicación de medidas para la conservación del suelo.

Méndez (2015) demostró que los sistemas de labranza de conservación cero tienen un efecto positivo sobre el contenido de materia orgánica en el suelo a través del tiempo.

\section{Conclusiones}

En las características morfológicas, el perfil bajo bosque es mullido húmico lavado, el perfil de cultivo presentó propiedades vérticas y en el pasto propiedades eslíticas. Además se observó contenido variable de carbonato en los tres perfiles.

$\mathrm{El} \mathrm{pH}$ va de ligeramente alcalino a alcalino, y es más alcalino en profundidad por la presencia de los carbonatos. La suma de bases cambiables es alta, sobre todo en la parte superior del perfil de pasto y cultivos, y muy alta en el horizonte superior del perfil de bosque. El contenido de materia orgánica es mayor en perfil de bosque en los horizontes superiores.

\section{Literatura Citada}

Arias, E.; Morales, A.; Ramis, E.; Fuentes, E.; Pérez, J.M.; Riverol, M.; Hernández, O.; Muñiz, O.; Aguilar, Y.

2010. Uso sostenible de los suelos en Cuba. Editorial Academia. La Habana, Cuba: Disponible en: http:// www.catalogo.bnjm.cu/cgi-bin/koha/opac-detail. pl?biblionumbe. Consultado: 7/Marzo/2018. Ciencia, Tecnología y Medio Ambiente. 2000. Programa Nacional de Lucha contra la Desertificación y la Sequía en la República de Cuba. CITMA. Ciudad de La Habana. Cuba. 167 p.

Cruz, J.; Nonato Rocha, S.; Camacho, J.; Castro, R.

2012. Spatial analysis of Physical atributes and organic carbon from yellow - red alfissol with sugarcane crop. Ciencias e Agrotecnologia Lavras, 34(2): 217-278.

Cruz, R.E.; Cruz, R.A.; Aguilera, G.L.I.; Norman, M.H.T.; Velázquez, R.A.; Nava, B.G.; Dendooven, L.; Reyes, R.B.G.

2012. Efecto en las características edáficas de un bosque templado por el cambio de uso de suelo. Terra Latinoamericana, 30(2): 189-197.
Domínguez, P.D.; Otero, M.A.; Ruiz, S.M.; Márquez, R.E.; Morejón, M.Y.

2006. Causas y efectos de la degradación del suelo en un agrosistema dedicado al cultivo del tabaco. Ciencia Tecnología y Medio Ambiente.8 (4). Disponible en: http://www.ciget.pinar. cu/Revista/No.2006-4/articulos/suelo\%20dunieski.pdf.

Gerasimova, M.I.; Khitrov, N.B.

2016. Morphological soil description for classifying soils and interpteting their genesis, Byulleten Pochvennogo instituta im. V.V. Dokuchaeva, 86: 8-16.

González, C.O.; Iglesias, C.E.; Herrera, S.M.

2009. Análisis de los factores que provocan compactación del suelo agrícola. Revista Ciencias Técnicas Agropecuarias, 18(2): 57-63.

Hernández, A., Bojórquez Serrano, J.I.; Morell Planes, F.; Cabrera Rodríguez, A.; Miguel, O.; García, A.; García Paredes, J.D.; Madueño Molina, A.; Nájera González, Y.O.

2010. Fundamentos de la estructura de suelos tropicales. Universidad Autónoma de Nayarit. México. 80 p. 
Hernández, A.; Paneque, J.; Pérez, J.M.; Mesa, A.; Bosch, D. y Fuentes, E.

1995.Metodología para la cartografía detallada y evaluación integral de los suelos. Instituto de Suelos y Dirección Nacional de Suelos y Fertilizantes. La Habana, Cuba. 53 p. Hernández, J.A.; Cabrera, R.A.; Borges, B.Y.; Vargas, B.D.; Bernal, F.A.; Morales, D.M.; \& Ascanio, G.M.O.

2013. Degradación de los suelos Ferralíticos Rojos Lixiviados y sus indicadores de la Llanura Roja de La Habana. Cultivos Tropicales, 34(3): 45-51.

Hernández, J.A.; Pérez, J.J.M.; Bosch, I.D. y Castro, S.N. 2015. Clasificación de los suelos de Cuba 2015. Ed. Ediciones INCA, 2015, Mayabeque, Cuba, 93 p.

Hernández, J.; Tirado, T.; Beltrán, H.

2014. Captura de carbono en los suelos. PÄDI Boletín Científico de Ciencias Básicas e Ingenierías del ICBI, 1(2). DOI: 10.29057/icbi.v1i2.506.

Iuss Working Group WRB.

2014. World Reference Base for soil resources 2014: international soil classification system for naming soils and creating legends for soil maps. (ser. World Soil Reports, no. ser. 106), Ed. Food and Agriculture Organization of the United Nations, Rome. $191 \mathrm{p}$.

Melo, V.F.; Orrutéa, A.G.; Motta, A.C.V.; Testoni, S.A.

2017. Land use and changes in soil morphology and physicalchemical properties in Southern Amazon. Rev Bras Cienc Solo, 41: e0170034

Morell, F.; Hernández, A.

2008. Degradación de las propiedades agrobiológicas de los suelos ferralíticos rojos lixiviados (nitisol ródico éutrico) por la influencia antrópica y su respuesta agroproductiva al mejoramiento. Agronomía trop., 58 (4):335-343.
Muñiz, O.

2017. Degradación de los Suelos. ForesightCuba. Disponible en: http://foresightcuba.com/degradacion Consultado: 12/11/2018

\section{MUNSELL}

2009. Munsell Soil Color Charts. Revised Washable Edition. GretagMacbeth. New Windsor, NY, US. 44 p.

Murray, R.; Nájera, O.; Orozco, B., María G.; Bojórquez, S.I. 2015. Cambios en carbono orgánico en suelos cambisoles, solonetz y arenosoles. Revista Iberoamericana de las Ciencias Biológicas y Agropecuarias, 4(8). Disponible en: http://www.ciba.org.mx/index.php/CIBA/article/view/31/98. Consultado: 12/11/2018

Navarro, A.; Figueroa, B.; Ordaz, V.; González, M.Y.; Félix V. 2000. Efecto de la labranza sobre la estructura del suelo, la germinación y el desarrollo del maíz y frijol. Terra, 18(1): 61-69.

Senjobi, B.A.; Ogunkunle, A.O.

2011. Effect of different land use types and their implications on land degradation and productivity in Ogun State, Nigeria. Journal of Agricultural Biotechnology and Sustainable Development, 3(1): 7-18.

Soil Survey Staff.

2010. Keys To Soil Taxonomy. Natural Resources Conservation Service y Agriculture Dept), $11^{\mathrm{a}}{ }^{\mathrm{e}} \mathrm{ed}$. Ed. Natural Resources Conservation Service. Washington, US. 344 p.

Torres, B.; Cortés, B.; Mejía, E.; Exebio, A.; Santos, A.; Delgadillo, Ma.

2003. Evaluación de la degradación de los suelos en la cuenca El Josefino, Jesús María, Jalisco. Terra Latinoamericana, 21(1): 117-126.

Walkley, A.; Black, I.A.

1934. An examination of the Degtjareff method for determining soil organic matter, and a proposed modification of the chromic acid titration method. Soil science, 37(1): 29-38. 
